PROCEEDINGS OF THE

AMERICAN MATHEMATICAL SOCIETY

Volume 125, Number 6, June 1997, Pages 1853-1859

S 0002-9939(97)04011-2

\title{
ON SIMULTANEOUS EXTENSION OF CONTINUOUS PARTIAL FUNCTIONS
}

\author{
HANS-PETER A. KÜNZI AND LEONID B. SHAPIRO
}

(Communicated by Franklin D. Tall)

\begin{abstract}
For a metric space $X$ let $\mathcal{C}_{v c}(X)$ (that is, the set of all graphs of real-valued continuous functions with a compact domain in $X$ ) be equipped with the Hausdorff metric induced by the hyperspace of nonempty closed subsets of $X \times \mathbf{R}$. It is shown that there exists a continuous mapping $\Phi: \mathcal{C}_{v c}(X) \rightarrow$ $\mathcal{C}_{b}(X)$ satisfying the following conditions:

(i) $\Phi(f) \mid \operatorname{dom} f=f$ for all partial functions $f$.

(ii) For every nonempty compact subset $K$ of $X, \Phi \mid \mathcal{C}_{b}(K): \mathcal{C}_{b}(K) \rightarrow \mathcal{C}_{b}(X)$ is a linear positive operator such that $\Phi\left(1_{K}\right)=1_{X}$.
\end{abstract}

\section{INTRODUCTION}

Let $X$ be a topological space. Recently V.V. Filippov [4] and his students studied the space $\mathcal{C}_{v}(X)$ of partial real-valued continuous functions with closed domains in $X$. (Let us mention that this space originates in $[5,6]$.):

Consider the set $\mathcal{C}_{v}(X)$ of all continuous functions $f: A \rightarrow \mathbf{R}$, where $A$ denotes a closed subspace of $X$, and identify each such function with its graph. Equip now $\mathcal{C}_{v}(X)$ with the topology induced by the Vietoris topology on the hyperspace $\exp (X \times \mathbf{R})$ of all nonempty closed subsets of $X \times \mathbf{R}$. In this paper we shall deal mainly with the subspace $\mathcal{C}_{v c}(X)$ of $\mathcal{C}_{v}(X)$ that consists of all partial continuous real-valued functions having a compact domain in $X$. Observe that for a metric space $X$ the space $\mathcal{C}_{v c}(X)$ is metrizable by the Hausdorff metric induced on $X \times \mathbf{R}$ [7].

For an arbitrary topological space we denote by $\exp _{c} X$ the set of nonempty compact subsets of $X$ equipped with the Vietoris topology. Furthermore let $\Pi$ : $\mathcal{C}_{v c}(X) \rightarrow \exp _{c} X$ be the natural projection. Note that for any $K \in \exp _{c} X$ the equality $\Pi^{-1}(K)=\mathcal{C}_{b}(K)$ holds. In this paper $\mathcal{C}_{b}(X)$ denotes the set of all bounded continuous real-valued functions $f$ on a topological space $X$ endowed with the topology given by the sup-norm $\|f\|_{\infty}=\sup _{x \in X}|f(x)|$.

Received by the editors December 16, 1995.

1991 Mathematics Subject Classification. Primary 54B20, 54C20, 54C35, 54C65, 54E15.

Key words and phrases. Extension of function, partial function, compact domain, Hausdorff metric, Lipschitzian function, probability measure.

The first author was partially working on this paper during his stay at the University of Łódź in 1995. He would like to thank his Polish colleagues for their hospitality.

During his visit to the University of Berne the second author was supported by the first author's grant 7GUPJ041377 from the Swiss National Science Foundation and by the International Science Foundation under grants NFU 000 and NFU 300. 
In the fifties J. Dugundji proved the following theorem.

Theorem 1 ([2]). Let $X$ be a metric space and $A$ a closed subspace of $X$ : Then there exists a linear operator $\Phi$ which makes correspond to each $f \in \mathcal{C}_{b}(A)$ an extension $\Phi(f) \in \mathcal{C}_{b}(X)$.

For a discussion of some related positive and negative results we refer the reader to $[11]$.

On the other hand E.N. Stepanova [10] proved recently that for a metric space $X$ it is possible to extend all elements of $\mathcal{C}_{v c}(X)$ simultaneously to $X$ in the following sense.

Theorem 2 ([10]). For any metrizable space $X$ there exists a continuous mapping $\alpha: \mathcal{C}_{v c}(X) \rightarrow \mathcal{C}_{b}(X)$ such that for any function $f, \alpha(f) \mid \operatorname{dom} f=f$ and $\sup _{x \in \operatorname{dom} f}|f(x)|=\sup _{x \in X}|\alpha(f)(x)|$.

She showed moreover that among the Hausdorff paracompact $p$-spaces only metrizable spaces have such a mapping $\alpha$.

In this paper we want to prove the following theorem.

Theorem 3. Let $X$ be a metrizable space. Then there exists a continuous mapping $\Phi: \mathcal{C}_{v c}(X) \rightarrow \mathcal{C}_{b}(X)$ that satisfies the following conditions:

(i) $\Phi(f) \mid \operatorname{dom} f=f$ for each partial function $f$;

(ii) for any $K \in \exp _{c} X$ the restriction $\Phi \mid \Pi^{-1}(K)$ is a linear positive operator such that $\Phi\left(1_{K}\right)=1_{X}$.

Observe that for compact domains our result generalizes simultaneously the theorems of Dugundji and Stepanova.

Remark 1. It is readily seen that each one-to-one (continuous) preimage of a space having an extension operator as described in Theorem 3 also admits such an operator. Therefore every one-to-one preimage of a metric space (i.e. a submetrizable space) admits an operator of this kind. Note that the class of one-to-one preimages of metric spaces contains all paracompact $\sigma$-spaces (compare [3, Exercise 5.5.7]). We deduce in particular that each stratifiable space admits an extension operator as described in Theorem 3. Observe however that the one-point-Lindelöfication of an uncountable discrete space admits such an operator, although it is not the one-to-one preimage of a metric space.

\section{Selections}

Our proof of Theorem 3 will be based on the following well-known selection theorem due to E. Michael [8, Theorem 3.2"]. Let $F: X \rightarrow Y$ be a lower semicontinuous multi-valued mapping from a paracompact Hausdorff space to a Banach space such that for each $x \in X, F(x)$ is a nonempty closed convex subset of $Y$. Then there exists a continuous mapping $f: X \rightarrow Y$ such that $f(x) \in F(x)$ whenever $x \in X$.

We recall that a multi-valued mapping $F: X \rightarrow Y$ is called lower semicontinuous provided that $\{x \in X: F(x) \cap U \neq \emptyset\}$ is open in $X$ whenever $U$ is open in $Y$.

For any metric space $(X, d)$, any $\delta>0$ and $A \subseteq X$ we set $B(A, \delta)=\{y \in X$ : $d(a, y)<\delta$ for some $a \in A\}$.

The proof of Theorem 3 consists of the verification of three lemmas. 
Lemma 1. Let $K$ and $L$ be compact subsets of a metric space $(X, d), r: X \rightarrow K a$ continuous mapping and $\epsilon$ a positive real number such that $\max _{x \in L} d(x, r(x))<\epsilon$. Then there exist a locally finite open cover $\gamma=\left\{V_{t}: t \in T\right\}$ of $X \backslash L$ and $\delta>0$ such that for any $t \in T$ with $V_{t} \cap B(L, \delta) \neq \emptyset$ we can choose $a_{t} \in K, b_{t} \in L$ and $y_{t} \in X \backslash L$ satisfying the following three conditions:

(i) $V_{t} \subseteq r^{-1}\left(B\left(a_{t}, 2 \epsilon\right)\right) \cap B\left(y_{t}, \frac{1}{4} d\left(y_{t}, L\right)\right)$;

(ii) $d\left(a_{t}, b_{t}\right)<2 \epsilon$; and

(iii) $d\left(y_{t}, b_{t}\right) \leq \frac{5}{4} d\left(y_{t}, L\right)$.

Proof. Note that $\mathcal{C}:=\left\{B\left(y, \frac{1}{4} d(y, L)\right) \cap r^{-1}(B(r(y), 2 \epsilon)): y \in X \backslash L\right\}$ is an open cover of $X \backslash L$. Since the subspace $X \backslash L$ of $X$ is paracompact, there is a locally finite open refinement $\gamma=\left\{V_{t}: t \in T\right\}$ of $\mathcal{C}$. We shall show that $\gamma$ satisfies the condition formulated in Lemma 1. Assume the contrary. Then for each $n \in \omega$ there is an element $V_{t_{n}} \in \gamma$ that hits $B\left(L, 2^{-n}\right)$, but we cannot find a triple $\left(a_{t_{n}}, b_{t_{n}}, y_{t_{n}}\right)$ as described in Lemma 1.

Fix $n \in \omega$. There is $y_{t_{n}} \in X \backslash L$ such that $V_{t_{n}} \subseteq B\left(y_{t_{n}}, \frac{1}{4} d\left(y_{t_{n}}, L\right)\right)$.

Next we want to verify that $d\left(y_{t_{n}}, L\right)<\frac{4}{3} \cdot 2^{-n}$. Indeed, let $y \in V_{t_{n}} \cap B\left(L, 2^{-n}\right)$. Thus $d\left(y_{t_{n}}, y\right)<\frac{1}{4} d\left(y_{t_{n}}, L\right)$. Let $z \in L$ be such that $d(y, z)=d(y, L)$. We have $d\left(y_{t_{n}}, L\right) \leq d\left(y_{t_{n}}, z\right) \leq d\left(y_{t_{n}}, y\right)+d(y, z)<\frac{1}{4} d\left(y_{t_{n}}, L\right)+2^{-n}$.

Therefore $\frac{3}{4} d\left(y_{t_{n}}, L\right)<2^{-n}$ and thus $d\left(y_{t_{n}}, L\right)<\frac{4}{3} \cdot 2^{-n}$.

It follows that $L \cup\left\{y_{t_{n}}: n \in \omega\right\}$ is a compact subset of $X$. Without loss of generality we can suppose that $\left(y_{t_{n}}\right)_{n \in \omega}$ is a convergent sequence and that $y:=\lim _{n \rightarrow \infty} y_{t_{n}} \in L$. For each $n \in \omega$ let $z_{t_{n}} \in L$ be such that $d\left(y_{t_{n}}, z_{t_{n}}\right)=$ $d\left(y_{t_{n}}, L\right)$. Then $y=\lim _{n \rightarrow \infty} z_{t_{n}}$, because $d\left(y_{t_{n}}, z_{t_{n}}\right) \rightarrow 0$. Since $d(y, r(y))<\epsilon$ and $r$ is continuous, there exists $\delta>0$ such that $\delta<\epsilon, B(y, \delta) \subseteq B(r(y), 2 \epsilon)$ and $r(B(y, \delta)) \subseteq B(r(y), 2 \epsilon)$.

Furthermore there exists $m \in \omega$ such that $B\left(y_{t_{m}}, \frac{1}{4} d\left(y_{t_{m}}, L\right)\right) \subseteq B(y, \delta)$ and $z_{t_{m}} \in B(y, \delta)$. Consequently for $V_{t_{m}}$ we can define the triple $\left(a_{t_{m}}=r(y), b_{t_{m}}=z_{t_{m}}\right.$, $\left.y_{t_{m}}\right)$. By our choice $V_{t_{m}} \subseteq B\left(y_{t_{m}}, \frac{1}{4} d\left(y_{t_{m}}, L\right)\right) \subseteq B(y, \delta) \subseteq r^{-1}(B(r(y), 2 \epsilon)) \stackrel{t_{m}}{=}$ $r^{-1}\left(B\left(a_{t_{m}}, 2 \epsilon\right)\right)$. Moreover $d\left(a_{t_{m}}, b_{t_{m}}\right)=d\left(r(y), z_{t_{m}}\right) \leq d(r(y), y)+d\left(y, z_{t_{m}}\right)<$ $\epsilon+\delta<2 \epsilon$. Finally $d\left(y_{t_{m}}, b_{t_{m}}\right)=d\left(y_{t_{m}}, z_{t_{m}}\right)=d\left(y_{t_{m}}, L\right) \leq \frac{5}{4} d\left(y_{t_{m}}, L\right)$.

Hence the defined triple satisfies conditions (i)-(iii) of Lemma 1 . We have reached a contradiction and conclude that the cover $\gamma$ possesses all the properties described in Lemma 1.

For a compact subset $K$ of a Banach space $X, \overline{c o} K$ will denote its closed convex hull in $X$. Note that $\overline{c o} K$ is compact, too (e.g. [9, Theorem 3.20]).

Let $\exp _{c} X$ be the hyperspace of nonempty compact subsets of $X$ equipped with the Hausdorff metric, which we shall denote by dist. Observe that for any sequence $\left(K_{n}\right)_{n \in \omega}$ converging to some point $K$ in $\exp _{c} X$, we have $\overline{\mathrm{co}} K_{n} \rightarrow \overline{\mathrm{co}} K$. It is known that the Hausdorff metric induces the Vietoris topology on $\exp _{c} X[7]$.

Lemma 2. Let $X$ be a Banach space and let $\mathcal{C}_{b}(X, X)$ be the Banach space of all bounded continuous $X$-valued functions on $X$ equipped with its sup-norm. Then there exists a continuous mapping $\mu: \exp _{c} X \rightarrow \mathcal{C}_{b}(X, X)$ such that for each $K \in$ $\exp _{c} X$ we have $\mu(K)(X) \subseteq \overline{\mathrm{co}} K$ and $\mu(K) \mid K=\mathrm{id}_{K}$.

Proof. Let $R: \exp _{c} X \rightarrow \mathcal{C}_{b}(X, X)$ be the multi-valued mapping defined by $K \mapsto$ $\left\{r \in \mathcal{C}_{b}(X, X): r(X) \subseteq \overline{\mathrm{co}} K\right.$ and $\left.r \mid K=\operatorname{id}_{K}\right\}$.

Note that for each $K \in \exp _{c} X, R(K)$ is a closed convex subset of $\mathcal{C}_{b}(X, X)$. It is nonempty by Dugundji's theorem [2, Theorem 4.1]. 
Since $\exp _{c} X$ is metrizable, it remains to be shown that $R$ is lower semi-continuous; then we can apply Michael's selection theorem in order to obtain the continuous mapping $\mu$ described in Lemma 2.

Let $U$ be an open subset of $\mathcal{C}_{b}(X, X)$ and let $K \in \exp _{c} X$ be such that $R(K) \cap U \neq$ $\emptyset$. If $K$ is not an interior point of $\left\{K \in \exp _{c} X: R(K) \cap U \neq \emptyset\right\}$, then there exists a sequence $\left(K_{n}\right)_{n \in \omega}$ in $\exp _{c} X$ converging to $K$ such that $R\left(K_{n}\right) \cap U=\emptyset$ whenever $n \in \omega$. Fix $r \in R(K) \cap U$ and let $\epsilon>0$ be such that $B(r, 2 \epsilon) \subseteq U$. For each $n \in \omega$ set $d_{n}=\max _{x \in K_{n}}\|r(x)-x\|$.

Since $r$ is continuous, $K_{n} \rightarrow K$ and $r \mid K=\mathrm{id}_{K}$, it is readily seen that the sequence $\left(d_{n}\right)_{n \in \omega}$ converges to 0 . Set $\delta=\frac{\epsilon}{4}$ and find $N \in \omega$ such that for any $n \in \omega$ with $n>N$ we have $\max \left\{\operatorname{dist}\left(\overline{\mathrm{co}} K, \overline{\mathrm{co}} K_{n}\right), d_{n}\right\}<\delta$.

Fix $n \in \omega$ such that $n>N$. We want to define $r_{n} \in R\left(K_{n}\right)$ such that

$$
\sup _{x \in X}\left\|r(x)-r_{n}(x)\right\| \leq \epsilon .
$$

To this end we are going to apply Lemma 1 . The role of $L$ is now played by $K_{n}$, the role of $K$ is played by $\overline{c o} K$ and the role of $\epsilon$ is played by $\delta$.

By Lemma 1 there exist a locally finite open cover $\gamma=\left\{V_{t}: t \in T\right\}$ of $X \backslash K_{n}$ and a neighborhood $V$ of $K_{n}$ in $X$ such that for each $V_{t}$ that hits $V$ we can find a triple $\left(a_{t}, b_{t}, y_{t}\right) \in \overline{\mathrm{co}} K \times K_{n} \times\left(X \backslash K_{n}\right)$ satisfying

(i) $V_{t} \subseteq r^{-1}\left(B\left(a_{t}, 2 \delta\right)\right) \cap B\left(y_{t}, \frac{1}{4}\left\|y_{t}-K_{n}\right\|\right)$;

(ii) $\left\|a_{t}-b_{t}\right\|<2 \delta$; and

(iii) $\left\|y_{t}-b_{t}\right\|<\frac{5}{4}\left\|y_{t}-K_{n}\right\|$.

If $V_{t} \cap V=\emptyset$, then in the light of the definition of the cover $\mathcal{C}$ in the proof of Lemma 1 we can choose $a_{t} \in \overline{\mathrm{co}} K$ such that $V_{t} \subseteq r^{-1}\left(B\left(a_{t}, 2 \delta\right)\right)$. Furthermore, since $\operatorname{dist}\left(\overline{\mathrm{co}} K, \overline{\mathrm{co}} K_{n}\right)<\delta$ we find $b_{t} \in \overline{\mathrm{co}} K_{n}$ such that $\left\|a_{t}-b_{t}\right\|<2 \delta$.

Now let $\left\{g_{t}: t \in T\right\}$ be a partition of unity on $X \backslash K_{n}$ subordinated to $\left\{V_{t}\right.$ : $t \in T\}$. Define $r_{n}: X \rightarrow X$ by $r_{n}(x)=x$ if $x \in K_{n}$ and $r_{n}(x)=\Sigma_{t \in T} g_{t}(x) b_{t}$ if $x \in X \backslash K_{n}$.

Note that $r_{n}(X) \subseteq \overline{\mathrm{co}} K_{n}$ and $r_{n} \mid K_{n}=\mathrm{id}_{K_{n}}$. Clearly $r_{n}$ is continuous at any $x \in X \backslash K_{n}$. Since inside $V \supseteq K_{n}$ our construction coincides with the one used by Dugundji as outlined by R. Engelking in [3, Exercise 4.5.20], we see that $r_{n}$ is also continuous at any $x \in K_{n}$.

Let us estimate $\sup _{x \in X}\left\|r(x)-r_{n}(x)\right\|$.

Case 1. Suppose that $x \in K_{n}$. Then $\left\|r(x)-r_{n}(x)\right\|=\|r(x)-x\| \leq d_{n}<\delta=\frac{\epsilon}{4}$.

Case 2. Suppose that $x \in X \backslash K_{n}$ and that $\left\{V_{t_{i}}: i=1, \ldots, m\right\}$ is the collection of all the members $V_{t} \in \gamma$ such that $x \in V_{t}$. Then $r_{n}(x)=\sum_{i=1}^{m} g_{t_{i}}(x) b_{t_{i}}$. Since $r\left(\bigcap_{i=1}^{m} V_{t_{i}}\right) \subseteq \bigcap_{i=1}^{m} B\left(a_{t_{i}}, 2 \delta\right)$, we have $\left\|r(x)-a_{t_{i}}\right\|<2 \delta$ whenever $i \leq m$.

Thus

$$
\begin{gathered}
\left\|r(x)-r_{n}(x)\right\|=\left\|r(x)-\sum_{i=1}^{m} g_{t_{i}}(x) b_{t_{i}}\right\| \\
\leq\left\|r(x)-\sum_{i=1}^{m} g_{t_{i}}(x) a_{t_{i}}\right\|+\left\|\sum_{i=1}^{m} g_{t_{i}}(x) a_{t_{i}}-\sum_{i=1}^{m} g_{t_{i}}(x) b_{t_{i}}\right\| \\
=\left\|\sum_{i=1}^{m} g_{t_{i}}(x) r(x)-\sum_{i=1}^{m} g_{t_{i}}(x) a_{t_{i}}\right\|+\left\|\sum_{i=1}^{m} g_{t_{i}}(x) a_{t_{i}}-\sum_{i=1}^{m} g_{t_{i}}(x) b_{t_{i}}\right\| \\
\leq\left(\sum_{i=1}^{m} g_{t_{i}}(x)\right)\left\|r(x)-a_{t_{i}}\right\|+\left(\sum_{i=1}^{m} g_{t_{i}}(x)\right)\left\|a_{t_{i}}-b_{t_{i}}\right\|<2 \delta+2 \delta=\epsilon .
\end{gathered}
$$

We conclude that $\sup _{x \in X}\left\|r(x)-r_{n}(x)\right\| \leq \epsilon$. It follows that $r_{n} \in R\left(K_{n}\right) \cap U$. We have reached a contradiction and deduce that $R: \exp _{c} X \rightarrow \mathcal{C}_{b}(X, X)$ is lower semi-continuous. 


\section{Measures}

Before proving Lemma 3 let us recall some facts established in [1]. Let $(X, d)$ be a metric space. Denote the $\sigma$-algebra of Borel sets of $X$ by $\mathcal{B}(X)$ and let $\mathcal{M}(X)$ be the set of all finite, real-valued, countably additive functions defined on $\mathcal{B}(X)$.

A bounded real-valued function $f$ on $X$ is called Lipschitzian if

$$
\|f\|_{L}=\sup \left\{\frac{|f(x)-f(y)|}{d(x, y)}: d(x, y) \neq 0\right\}<\infty .
$$

On the set $B L(X, d)$ of all such functions a norm is defined by setting $\|f\|_{B L}=$ $\|f\|_{\infty}+\|f\|_{L}$. Then $\left(B L(X, d),\|\cdot\|_{B L}\right)$ is a Banach algebra according to [1].

We shall denote its dual space by $B L(X, d)^{*}$ and the corresponding norm by $\|T\|_{B L}^{*}=\sup \left\{|T(f)|:\|f\|_{B L}=1\right\}$. By $\mu \in \mathcal{M}(X) \mapsto \mu(f)=\int f d \mu \in B L(X, d)^{*}$ an injection of $\mathcal{M}(X)$ into $B L(X, d)^{*}$ is defined.

In this way we can consider $\mathcal{M}(X)$ a subset of $B L(X, d)^{*}$. The topology induced on $\mathcal{M}(X)$ by the norm on $B L(X, d)^{*}$ will be denoted by $T B L^{*}$. The weak ${ }^{*}$ topology $T W^{*}$ on $\mathcal{M}(X)$ is determined by $\mu_{n} \rightarrow \mu$ iff $\int f d \mu_{n} \rightarrow \int f d \mu$ whenever $f$ in $\mathcal{C}_{b}(X)$. It coincides with $T B L^{*}$ on the set $\mathcal{M}_{s}^{+}(X)$ (of nonnegative measures with separable supports) [1, Theorem 18].

Let us denote by $\mathcal{P}_{c}(X)$ the space of the probability measures with compact supports on $X$. Then $\mathcal{P}_{c}(X) \subseteq \mathcal{M}_{s}^{+}(X)$ and the weak* topology on $\mathcal{P}_{c}(X)$ coincides with the topology induced on $\mathcal{P}_{c}(X)$ by $\|\cdot\|_{B L}^{*}$.

It is known that $x \mapsto \delta_{x}$ defines a topological embedding of $X$ into $B L(X, d)^{*}$. Here, as usual, $\delta_{x}$ denotes the Dirac measure.

Lemma 3. Let $\mu: \exp _{c} X \rightarrow \mathcal{C}_{b}\left(X, B L(X, d)^{*}\right)$ be a continuous mapping such that for each $K \in \exp _{c} X$ we have that $\mu(K)(X) \subseteq \mathcal{P}(K)$ (where $\mathcal{P}(K)$ denotes the space of probability measures on $K$ ) and $\mu(K) \mid K=\mathrm{id}_{K}$ (modulo the identification $\left.x \mapsto \delta_{x}\right)$.

Then the mapping $\Phi: \mathcal{C}_{v c}(X) \rightarrow \mathcal{C}_{b}(X)$ given by the formula

$$
\Phi(f)(x)=\int f d \mu(\operatorname{dom} f)(x)
$$

for every partial function $f$ and every $x \in X$ satisfies the conditions of Theorem 3 .

Remark 2. Let us note that Theorem 3 is an immediate consequence of Lemma 2 (applied to the Banach space $B L(X, d)^{*}$ ) and Lemma 3, because $\mathcal{P}(K)$ is a closed convex set in $B L(X, d)^{*}$.

Proof of Lemma 3. Note first that $\Phi(f)$ is an extension of $f$, since for any $x \in \operatorname{dom} f$ we have $\mu(\operatorname{dom} f)(x)=\delta_{x}$ and therefore $\Phi(f)(x)=\int f d \delta_{x}=f(x)$.

Clearly for any $K \in \exp _{c} X, \Phi: \mathcal{C}_{b}(K) \rightarrow \mathcal{C}_{b}(X)$ is a linear positive operator such that $\sup _{x \in X}|\Phi(f)(x)|=\sup _{x \in \operatorname{dom} f}|f(x)|$ and $\Phi\left(1_{K}\right)=1_{X}$. Furthermore $\Phi(f)$ is continuous, since $\mathcal{P}(\operatorname{dom} f)$ is endowed with the weak* topology.

It remains to check the continuity of $\Phi$. To this end suppose that $f_{n} \rightarrow f$ in $\mathcal{C}_{v c}(X), K=\operatorname{dom} f$ and $K_{n}=\operatorname{dom} f_{n}$ whenever $n \in \omega$. In the following the Hausdorff metric on $\mathcal{C}_{v c}(X)$ will be denoted by dist .

Obviously $K_{n} \rightarrow K$ in $\exp _{c} X$, since $f_{n} \rightarrow f$. Therefore $\widetilde{K}=K \cup \bigcup\left\{K_{n}: n \in \omega\right\}$ is compact in $X$ [7, Theorem 2.5]. Let us fix an arbitrary continuous real-valued extension $\tilde{f}$ of $f$ to $X$. We can consider $\tilde{f}$ a continuous real-valued function defined on $\mathcal{P}(\widetilde{K})$ by setting $\widetilde{f}(\mu)=\int \widetilde{f} d \mu$ whenever $\mu \in \mathcal{P}(\widetilde{K})$. Note that $\widetilde{f}$ is uniformly continuous with respect to the norm $\|\cdot\|_{B L}^{*}$ restricted to $\mathcal{P}(\widetilde{K})$. 
Define the partial continuous functions $g_{n}: K_{n} \rightarrow \mathbf{R}$ by setting $g_{n}=\widetilde{f} \mid K_{n}$ where $n \in \omega$. It follows that $g_{n} \rightarrow f$ in $\mathcal{C}_{v c}(X)$, because $K_{n} \rightarrow K\left(\right.$ in $\left.\exp _{c} X\right)$. Then $\operatorname{dist}\left(f_{n}, g_{n}\right) \rightarrow 0$ in the metric space $\mathcal{C}_{v c}(X)$. This implies that

$$
\max _{x \in K_{n}}\left|f_{n}(x)-g_{n}(x)\right|=\max _{x \in K_{n}}\left|f_{n}(x)-\widetilde{f}(x)\right| \rightarrow 0,
$$

as it is readily verified.

Now let $\epsilon>0$. We want to find $N \in \omega$ such that for all $n \in \omega$ with $n>N$ and all $x \in X,\left|\Phi(f)(x)-\Phi\left(f_{n}\right)(x)\right|<\epsilon$.

By uniform continuity of $\widetilde{f}$ on $\mathcal{P}(\widetilde{K})$ we first find $\delta>0$ such that if $\mu, \nu \in \mathcal{P}(\widetilde{K})$ and $\|\mu-\nu\|_{B L}^{*}<\delta$, then $\left|\int \widetilde{f} d \mu-\int \widetilde{f} d \nu\right|<\frac{\epsilon}{2}$.

Furthermore there exists $N_{1} \in \omega$ such that for all $n \in \omega$ with $n>N_{1}$ we have $\max _{x \in K_{n}}\left|f_{n}(x)-\widetilde{f}(x)\right|<\frac{\epsilon}{2}$. Moreover there exists $N_{2} \in \omega$ such that for all $n \in \omega$ with $n>N_{2}$ and all $x \in X$ we have that $\left\|\mu(K)(x)-\mu\left(K_{n}\right)(x)\right\|_{B L}^{*}<\delta$, because $\mu$ is continuous.

Let $N=\max \left\{N_{1}, N_{2}\right\}$. For all $n \in \omega$ such that $n>N$ and all $x \in X$, we obtain finally:

$$
\begin{gathered}
\left|\Phi(f)(x)-\Phi\left(f_{n}\right)(x)\right|=\left|\int f d \mu(K)(x)-\int f_{n} d \mu\left(K_{n}\right)(x)\right| \\
\leq\left|\int f d \mu(K)(x)-\int \tilde{f} d \mu\left(K_{n}\right)(x)\right|+\left|\int \widetilde{f} d \mu\left(K_{n}\right)(x)-\int f_{n} d \mu\left(K_{n}\right)(x)\right| \\
=\left|\int \tilde{f} d \mu(K)(x)-\int \tilde{f} d \mu\left(K_{n}\right)(x)\right|+\left|\int \tilde{f} d \mu\left(K_{n}\right)(x)-\int f_{n} d \mu\left(K_{n}\right)(x)\right| \\
<\frac{\epsilon}{2}+\max _{x \in K_{n}}\left|\tilde{f}(x)-f_{n}(x)\right|<\frac{\epsilon}{2}+\frac{\epsilon}{2}=\epsilon .
\end{gathered}
$$

We have shown that $\Phi$ is continuous.

Remark 3. Let us finally remark that it is possible to obtain a variant of Theorem 3 by replacing the reals by an arbitrary Banach space over the reals. In fact we can prove the following result.

If $E$ is a Hausdorff sequentially complete locally convex topological vector space over the reals, then there exists a continuous mapping $\Phi: \mathcal{C}_{v c}(X, E) \rightarrow C(X, E)$ (where $C(X, E)$ is equipped with the topology of uniform convergence) that satisfies the following conditions:

(i) $\Phi(f) \mid \operatorname{dom} f=f$ for any partial function $f \in C_{v c}(X, E)$;

(ii) range $(\Phi(f)) \subseteq \overline{\mathrm{co}}($ range $f)$;

(iii) for any $K \in \exp _{c} X$ the restriction $\Phi \mid \Pi^{-1}(K)$ is a linear operator from $C(K, E)$ into $C(X, E)$.

Indeed, according to Lemmas 2 and 3 let $\mu: \exp _{c} X \rightarrow \mathcal{C}_{b}\left(X, B L(X, d)^{*}\right)$ be a continuous mapping such that for each $K \in \exp _{c} X$ we have that $\mu(K)(X) \subseteq \mathcal{P}(K)$ (where $\mathcal{P}(K)$ denotes the space of probability measures on $K$ ) and $\mu(K) \mid K=\operatorname{id}_{K}$ (modulo the identification $x \mapsto \delta_{x}$ ).

First we assume that $E$ is a Banach space. Then the formula $\Phi(f)(x)=$ $\int f d \mu(\operatorname{dom} f)(x)$ gives the desired mapping. In order to check the statement observe that $\Phi(f)$ is a continuous mapping from $X$ into $E$ : Since if $x_{n} \rightarrow x$ in $X$, then $\int f d \mu(\operatorname{dom} f)\left(x_{n}\right)$ weakly converges to $\int f d \mu(\operatorname{dom} f)(x)$; but

$$
\int f d \mu(\operatorname{dom} f)\left(x_{n}\right) \in \overline{\mathrm{co}}(\text { range } f)
$$


and $\overline{\mathrm{co}}($ range $f)$ is a compact space. Therefore weak convergence coincides with the usual convergence in $E$. The proof of the continuity of $\Phi$ is the same as in the proof of Lemma 3 (instead of $\mathbf{R}$ we are working with $E$ ).

Now suppose that $E$ is a Hausdorff sequentially complete LCTVS. Then we can assume that $E$ is a subspace of a product of Banach spaces $\prod\left\{E_{\alpha}: \alpha \in A\right\}$.

Hence we set $\Phi(f)(x):=\int f d \mu(\operatorname{dom} f)(x)=\left\{\int \operatorname{pr}_{\alpha} \circ f d \mu(\operatorname{dom} f)(x): \alpha \in\right.$ $A\}$. Let us note that according to sequential completeness of $E, \int f d \mu(\operatorname{dom} f)(x)$ belongs to $E$ : Clearly $\mu(\operatorname{dom} f)(x)$ is a weak limit of a sequence $\left\{\mu_{n}: n \in \omega\right\}$ where supp $\mu_{n} \subseteq \operatorname{dom} f$ and $\left|\operatorname{supp} \mu_{n}\right|<\omega$. Consequently $\int f d \mu_{n} \in \operatorname{co}($ range $f)$ and $\left\{\int f d \mu_{n}: n \in \omega\right\}$ is a Cauchy sequence in $E$. Thus $\int f d \mu(\operatorname{dom} f)(x) \in E$ as a limit of a Cauchy sequence.

The continuity of $\Phi$ follows from the continuity of the mappings $\Phi_{\alpha}$, where $\left(\Phi_{\alpha}(f)\right)(x)=\int \operatorname{pr}_{\alpha} \circ f d \mu(\operatorname{dom} f)(x)$.

\section{REFERENCES}

[1] R.M. Dudley, Convergence of Baire measures, Studia Math. 27 (1966), 251-268. MR 50:7466

[2] J. Dugundji, An extension of Tietze's theorem, Pacific J. Math. 1 (1951), 353-367. MR 13:373c

[3] R. Engelking, General Topology, Heldermann, Berlin, 1989.

[4] V.V. Filippov, Topological structure of solution spaces of ordinary differential equations, in Russian, Uspekhi Mat. Nauk 48 (1993), 103-154. MR 94f:34008

[5] K. Kuratowski, Sur l'espace des fonctions partielles, Ann. Mat. Pura Appl. 40 (1955), 61-67. MR 17:650b

[6] K. Kuratowski, Sur une méthode de métrisation complète de certains espaces d'ensembles compacts, Fund. Math. 43 (1956), 114-138. MR 18:58a

[7] E. Michael, Topologies on spaces of subsets, Trans. Amer. Math. Soc. 71 (1951), 152-182. MR 13:54f

[8] E. Michael, Continuous selections, I, Ann. Math. 63 (1956), 361-382. MR 17:990e

[9] W. Rudin, Functional Analysis, McGraw-Hill, New York, 1991. MR 92k:46001

[10] E.N. Stepanova, Extension of continuous functions and metrizability of paracompact pspaces (in Russian), Mat. Zametki 53 (1993), 92-101; translation in Math. Notes 53 (1993), 308-314. MR 94k:54031

[11] E.K. van Douwen, Simultaneous linear extension of continuous functions, Gen. Topology Appl. 5 (1975), 297-319. MR 52:1612

Department of Mathematics, University of Berne, Sidlerstrasse 5, CH-3012 Berne, SWITZERLAND

E-mail address: kunzi@math-stat.unibe.ch

Department of Mathematics, Academy of Labor and Social Relations, Lobachevskogo 90, 117454 Moscow, Russia

E-mail address: lshapiro@glas.apc.org 